\title{
Correction to: Impact of depth of clinical response on outcomes of acute myeloid leukemia patients in first complete remission who undergo allogeneic hematopoietic cell transplantation
}

\author{
Mary-Elizabeth Percival (1) - Hai-Lin Wang - Mei-Jie Zhang - Wael Saber - Marcos de Lima (1) - Mark Litzow • \\ Partow Kebriaei (D) Hisham Abdel-Azim · Kehinde Adekola - Mahmoud Aljurf • Ulrike Bacher · Sherif M. Badawy · \\ Amer Beitinjaneh - Nelli Bejanyan (1D) Vijaya Bhatt (10) Michael Byrne · Jean-Yves Cahn · Paul Castillo (1) • \\ Nelson Chao - Saurabh Chhabra - Edward Copelan - Corey Cutler - Zachariah DeFilipp (1) Ajoy Dias · \\ Miguel Angel Diaz • Elihu Estey · Nosha Farhadfar · Haydar A. Frangoul · César O. Freytes · Robert Peter Gale • \\ Siddhartha Ganguly • Lohith Gowda - Michael Grunwald (1) - Nasheed Hossain (1) - Rammurti T. Kamble • \\ Christopher G. Kanakry • Ankit Kansagra $(\mathbb{D}) \cdot$ Mohamed A. Kharfan-Dabaja $(\mathbb{D} \cdot$ Maxwell Krem • \\ Hillard M. Lazarus (1) - Jong Wook Lee · Jane L. Liesveld • Richard Lin $(\mathbb{D} \cdot$ Hongtao Liu • Joseph McGuirk (D) \\ Reinhold Munker - Hemant S. Murthy - Sunita Nathan (1) - Taiga Nishihori (1) - Richard F. Olsson - Neil Palmisiano • \\ Jakob R. Passweg • Tim Prestidge (1) - Olov Ringdén (1) - David A. Rizzieri - Witold B. Rybka (1) Mary Lynn Savoie • \\ Kirk R. Schultz $(\mathbb{D} \cdot$ Sachiko Seo 1 - Akshay Sharma 1 - Melhem Solh $(1) \cdot$ Roger Strair - Marjolein van der Poel • \\ Leo F. Verdonck · Jean A. Yared (1) - Daniel Weisdorf (1) - Brenda M. Sandmaier (1)
}

(c) The Author(s), under exclusive licence to Springer Nature Limited 2021

Correction to: Bone Marrow Transplantation https://doi.org/10.1038/s41409-021-01261-6; Article published online 16 April 2021
Unfortunately, in Acknowledgement part needs to be removed the funding number OT3HL147741.

The original article has been corrected. 